\title{
A New Scheme on Synchronization of Commensurate Fractional-Order Chaotic Systems Based on Lyapunov Equation
}

\author{
Hua Wang, Hang-Feng Liang, Peng Zan, and Zhong-Hua Miao \\ Shanghai Key Laboratory of Power Station Automation Technology, School of Mechatronics Engineering and Automation, \\ Shanghai University, Shanghai 200072, China
}

Correspondence should be addressed to Zhong-Hua Miao; zhhmiao@shu.edu.cn

Received 12 January 2016; Revised 29 June 2016; Accepted 8 August 2016

Academic Editor: Francisco Gordillo

Copyright (C) 2016 Hua Wang et al. This is an open access article distributed under the Creative Commons Attribution License, which permits unrestricted use, distribution, and reproduction in any medium, provided the original work is properly cited.

\begin{abstract}
This paper proposes a new fractional-order approach for synchronization of a class of fractional-order chaotic systems in the presence of model uncertainties and external disturbances. A simple but practical method to synchronize many familiar fractionalorder chaotic systems has been put forward. A new theorem is proposed for a class of cascade fractional-order systems and it is applied in chaos synchronization. Combined with the fact that the states of the fractional chaotic systems are bounded, many coupled items can be taken as zero items. Then, the whole system can be simplified greatly and a simpler controller can be derived. Finally, the validity of the presented scheme is illustrated by numerical simulations of the fractional-order unified system.
\end{abstract}

\section{Introduction}

Fractional calculus, with more than 300-year-old history, is generalization of ordinary differentiation and integration to arbitrary order. Until the recent decades, the fractional calculus attracted attention of researchers in various fields [1-5]. Many systems in physics and engineering have been found to be effectively modeled as fractional-order systems [6-8]. Fractional-order systems could also behave chaotically. Recently, it has been demonstrated that some fractionalorder differential systems, including fractional-order Lü system, fractional-order Chen system, fractional-order coupled dynamos system, fractional-order Liu system, and fractionalorder unified system [9-13] exhibit chaotic behaviors.

Integer order chaotic systems have been extensively studied for many decades [14, 15], whereas their fractionalorder counterparts have just recently been investigated. An extensive survey of various fractional-order chaotic systems can be found in [4]. Chaos control of fractional-order chaotic systems has received a lot of considerations of researchers. However, compared to integer order chaotic systems, the research results in this field are relatively rare. For the limitation of the available theoretical tools, a number of research works mainly focus on integer order chaotic systems.

Chaos control based on stability theories for linear fractional-order systems has been extensively proposed in $[16,17]$. However, this approach provides only local stability. Active control is another approach that can be found in the literature $[18,19]$. The approach made use of a nonlinear control law to cancel nonlinearities in the control system. Based on the stability theory for linear fractionalorder systems, a linear control law was designed to stabilize the linearized system. Reference [20] presented LMI-based stabilization method for fractional-order chaotic systems. The method required that the systems must be transformable into a linear interval fractional-order system. In $[21,22]$, the authors proposed a sliding mode control approach to realize chaos control. By adopting a fractional-order sliding surface, the stability can be obtained via the Lyapunov stability method. However, the fractional-order sliding surface might be difficult to implement. Moreover, discontinuous control signals could induce some undesired behavior.

Many of the above controllers are nonlinear. From a practical point of view, to design a simple linear controller 
by a special strategy is more valuable. However, to our best knowledge, there is a limitation of the available theoretical tools that can be used for nonlinear fractional-order systems. Therefore, designing a linear controller for fractional-order chaotic systems has still remained as an open and challenging problem.

Motivated by the above discussions, in this paper, based on fractional-order Lyapunov stability theory, a new strategy is proposed to synchronize a class of fractional-order chaotic systems in the presence of model uncertainties and external disturbances. Then, a simple linear controller with low dimensions is designed via the proposed new strategy. Compared with the control approach in [20], only two simple and linear feedback controllers are designed to achieve synchronization. Compared with the control approach in $[21,22]$, it can be implemented easily.

The rest of this paper is organized as follows: in Section 2, some preliminaries and main results are presented. In Section 3, we give the famous fractional-order unified chaotic systems to illustrate that it can be transformed to our cascade form for further simple design. Experimental analyses are presented in Section 4. Finally, conclusions are given in Section 5.

\section{Preliminaries and Main Results}

In this section, first some basic definitions and lemmas are briefly presented. Then a useful theorem is put forward to be utilized later to design a robust linear control law.

Definition 1. The definition of fractional integral is described by

$$
{ }_{t_{0}} D_{t}^{-\alpha} f(t)=\frac{1}{\Gamma(\alpha)} \int_{t_{0}}^{t}(t-\tau)^{\alpha-1} f(\tau) d \tau, \quad \alpha>0,
$$

where $\Gamma(\cdot)$ is the well-known Gamma function.

Definition 2. The Riemann-Liouville derivative is defined as

$$
D_{t}^{q} f(t)=D_{t_{0}}^{m} D_{t}^{q-m} f(t), \quad q \in[m-1, m),
$$

where $m \in Z^{+}, D_{t}^{m}$ is the classical $m$-order derivative.

In the rest of the paper, $D_{t}^{q}$ is used to denote the RiemannLiouville derivative of order $q$.

Lemma 3. Assume $f(t)$ is bounded and $\lim _{t \rightarrow \infty} g(t)=0$; then $\lim _{t \rightarrow \infty}[f(t) g(t)]=0$.

Proof. Since $f(t)$ is bounded, then there exists a number $M>$ 0 such that $|f(t)|<M$ for all $t$.

$\forall \varepsilon>0$, since $\lim _{t \rightarrow \infty} g(t)=0$, then, for $\varepsilon_{1}=\varepsilon / M>0, \exists \delta$ such that

$$
|g(t)|<\varepsilon_{1}=\frac{\varepsilon}{M}, \quad \text { when } t>\delta
$$

Then, when $t>\delta$

$$
|f(t) g(t)| \leq|f(t)||g(t)|<M * \varepsilon_{1}=\varepsilon .
$$

This means $\lim _{t \rightarrow \infty}[f(t) g(t)]=0$. Here the proof is complete.

Lemma 4 (see [23]). Let $A \in R^{n \times n}$ be a real matrix. Then, a necessary and sufficient condition for the asymptotical stability of $D^{\alpha} x(t)=A x(t)$ is

$$
|\arg (\lambda(A))|>\frac{\alpha \pi}{2}
$$

where $0<\alpha<1, \lambda(A)$ is the spectrum of all eigenvalues of $A$.

In this paper, we consider the case of the synchronization of two commensurate fractional-order chaotic systems. The master system is as follows:

$$
D^{q} x=A(x) x
$$

where $x=\left(x_{1}, x_{2}, \ldots, x_{n}\right)^{T} \in R^{n}$ is the state vector, $A(x)$ is the parametric coefficient matrix of states $x$, and $0<q \leq 1$ is the fractional commensurate order. The slave system is

$$
D^{q} \widehat{x}=A(\widehat{x}) \hat{x}+u(t),
$$

where $u(t)$ is the controller to be designed. Define $e(t)=$ $\widehat{x}(t)-x(t)$. We can get the error system:

$$
D^{q} e=F(e, x)+u(e, x),
$$

where $F(e, x)=A(\hat{x}) \hat{x}-A(x) x$. The synchronization problem can be transformed to design a controller $u$ such that $\lim _{t \rightarrow \infty}\|e(t)\|=0$.

For further discussion, a useful theorem is presented. Consider a class of cascade-connected system described by

$$
\begin{aligned}
D^{q} x_{1} & =f\left(x_{1}\right) \\
D^{q}\left(x_{1}, x_{2}\right) & =A\left(x_{1}, x_{2}\right) x_{2}+B\left(x_{1}, x_{2}\right) g\left(x_{1}\right),
\end{aligned}
$$

where $x_{1} \in R^{n}, x_{2} \in R^{m}, f(0)=0$, and $g(0)=0 . f\left(x_{1}\right)$ and $g\left(x_{1}\right)$ are both $C^{1}$ vector fields. $A\left(x_{1}, x_{2}\right)$ and $B\left(x_{1}, x_{2}\right)$ are $C^{m}$ and $C^{n}$ coefficient matrix, respectively.

Theorem 5. If

(1) the subsystem $D^{q} x_{1}=f\left(x_{1}\right)$ is globally asymptotically stable at $x_{1}=0$,

(2) $B\left(x_{1}, x_{2}\right)$ is a bounded matrix and $\lim _{t \rightarrow \infty} g\left(x_{1}\right)=0$,

(3) $A\left(x_{1}, x_{2}\right)$ is a matrix with the following structure:

$A\left(x_{1}, x_{2}\right)$

$$
=\left(\begin{array}{cccc}
A_{11}(\cdot) & A_{12}(\cdot) & \ldots & A_{1 n}(\cdot) \\
A_{21}(\cdot) & A_{22}(\cdot) & \ddots & \vdots \\
\vdots & \vdots & \ddots & A_{n-1 n}(\cdot) \\
A_{n 1}(\cdot) & \ldots & A_{n n-1}(\cdot) & A_{n n}(\cdot)
\end{array}\right) \text {, }
$$

where $\left(A_{i j}(\cdot) \leq 0\right), i=j,\left(A_{i j}(\cdot)=-A_{j i}(\cdot)\right), i \neq j$, then, system (9) is globally asymptotically stable at the equilibrium $\left(x_{1}, x_{2}\right)=(0,0)$. 
Proof. Since the subsystem

$$
D^{q} x_{1}=f\left(x_{1}\right)
$$

is already globally asymptotically stable at $x_{1}=0$, we only need to consider the subsystem

$$
D^{q}\left(x_{1}, x_{2}\right)=A\left(x_{1}, x_{2}\right) x_{2}+B\left(x_{1}, x_{2}\right) g\left(x_{1}\right) .
$$

From assumption (2) we know that $\lim _{t \rightarrow \infty} g\left(x_{1}\right)=0$. Consider the fact that $B\left(x_{1}, x_{2}\right)$ is a bounded matrix. From Lemma 3 we know that

$$
\lim _{t \rightarrow \infty}\left[B\left(x_{1}, x_{2}\right) g\left(x_{1}\right)\right]=0 .
$$

This means that the second item in the system

$$
D^{q}\left(x_{1}, x_{2}\right)=A\left(x_{1}, x_{2}\right) x_{2}+B\left(x_{1}, x_{2}\right) g\left(x_{1}\right)
$$

can be neglected when $t \rightarrow \infty$. To study the asymptotical stability problem of system (9), we only need to consider the following system:

$$
D^{q}\left(x_{1}, x_{2}\right)=A\left(x_{1}, x_{2}\right) x_{2} .
$$

Here, we take three steps to illustrate that system (15) is globally asymptotically stable at the equilibrium $\left(x_{1}, x_{2}\right)=$ $(0,0)$.

Firstly, let us consider the following equation:

$$
A\left(x_{1}, x_{2}\right) \xi=\lambda \xi
$$

where $\lambda$ is one of the eigenvalues of $A\left(x_{1}, x_{2}\right)$ and $\xi$ is a nonzero eigenvector of $\lambda$. Take the conjugate transpose on either side of (16) and we can get

$$
{\overline{\left(A\left(x_{1}, x_{2}\right) \xi\right)}}^{T}=\bar{\lambda} \xi^{H}
$$

Combine (16) with (17) and we can get

$$
\xi^{H}\left(P A\left(x_{1}, x_{2}\right)+\left(A\left(x_{1}, x_{2}\right)\right)^{H} P\right) \xi=(\lambda+\bar{\lambda}) \xi^{H} P \xi .
$$

Secondly, because $A\left(x_{1}, x_{2}\right)$ has the structure

$$
\begin{aligned}
& A\left(x_{1}, x_{2}\right) \\
& =\left(\begin{array}{cccc}
A_{11}(\cdot) & A_{12}(\cdot) & \ldots & A_{1 n}(\cdot) \\
A_{21}(\cdot) & A_{22}(\cdot) & \ddots & \vdots \\
\vdots & \vdots & \ddots & A_{n-1 n}(\cdot) \\
A_{n 1}(\cdot) & \ldots & A_{n n-1}(\cdot) & A_{n n}(\cdot)
\end{array}\right) \\
& =\left(\begin{array}{cccc}
A_{11}(\cdot) & A_{12}(\cdot) & \ldots & A_{1 n}(\cdot) \\
-A_{12}(\cdot) & A_{22}(\cdot) & \ddots & \vdots \\
\vdots & \ddots & \ddots & A_{n-1 n}(\cdot) \\
-A_{1 n}(\cdot) & \ldots & -A_{n-1 n}(\cdot) & A_{n n}(\cdot)
\end{array}\right),
\end{aligned}
$$

we can know that the matrix $A\left(x_{1}, x_{2}\right)$ satisfies the continuous Lyapunov equation

$$
A\left(x_{1}, x_{2}\right) P+P A\left(x_{1}, x_{2}\right)^{H}=-Q
$$

where $P=I$ is the real symmetric identity matrix and

$$
Q=\left(\begin{array}{cccc}
-2 A_{11} & & & \\
& -2 A_{22} & & \\
& & \ddots & \\
& & & -2 A_{n n}
\end{array}\right)
$$

is a Hermitian matrix. Moreover, we can have

$$
\begin{aligned}
& A\left(x_{1}, x_{2}\right) P+P A\left(x_{1}, x_{2}\right)^{H} \\
& \quad=\left(A\left(x_{1}, x_{2}\right) P+P A\left(x_{1}, x_{2}\right)^{H}\right)^{H} .
\end{aligned}
$$

Namely, $A\left(x_{1}, x_{2}\right) P+P\left(A\left(x_{1}, x_{2}\right)\right)^{H}$ is also a Hermitian matrix.

Lastly, according to the properties of positive definite and negative semidefinite matrix, we can get two inequalities

$$
\begin{gathered}
\xi^{H}\left(P A\left(x_{1}, x_{2}\right)+\left(A\left(x_{1}, x_{2}\right)\right)^{H} P\right) \xi=\xi^{H}(-Q) \xi \leq 0 \\
\xi^{H} P \xi>0 .
\end{gathered}
$$

Consequently, combining (18), we can have

$$
(\lambda+\bar{\lambda})=\frac{\xi^{H}(-Q) \xi}{\left(\xi^{H} P \xi\right)} \leq 0 .
$$

Obviously, any eigenvalue $\lambda$ of the coefficient matrix $A\left(x_{1}, x_{2}\right)$ satisfies the following inequality:

$$
|\arg (\lambda)| \geq \frac{\pi}{2}>\frac{\alpha \pi}{2} \quad(\alpha<1)
$$

From Lemma 4, we know that system (15) is globally asymptotically stable at the equilibrium $\left(x_{1}, x_{2}\right)=(0,0)$. This completes the proof.

\section{Illustrative Example}

In the first part of section, the fractional-order unified chaotic system and its synchronization are transformed to our cascade form and a simpler controller is derived. Then its robustness analysis will be introduced.

3.1. Fractional-Order Unified Chaotic System and Its Synchronization. Similar to the classical unified chaotic system, the fractional-order unified system could be considered as the system that bridges the gap among the fractionalorder Lorenz system, the fractional-order Lü system, and 


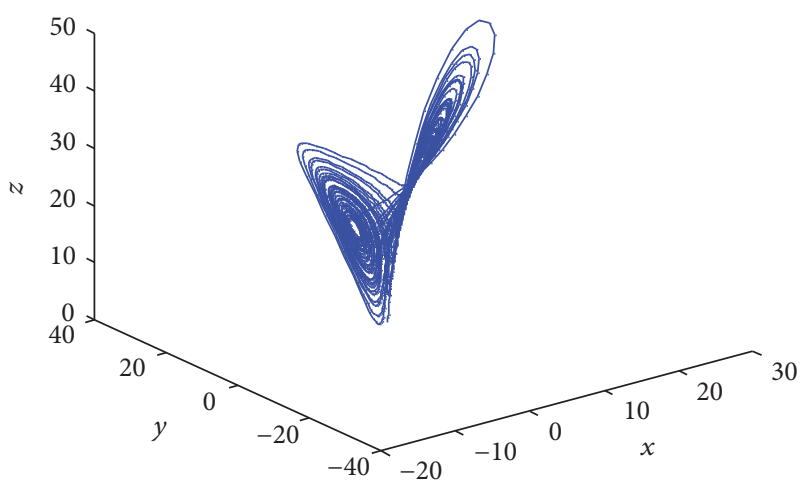

FIGURE 1: The fractional-order unified chaotic system with $q_{1}=q_{2}=$ $q_{3}=0.95$ and $\alpha=0.2$.

the fractional-order Chen system. The fractional-order unified chaotic system [13] is described by

$$
\begin{aligned}
& \frac{d^{q_{1}} x}{d t^{q_{1}}}=(25 \alpha+10)(y-x) \\
& \frac{d^{q_{2}} y}{d t^{q_{2}}}=(28-35 \alpha) x-x z+(29 \alpha-1) y \\
& \frac{d^{q_{3}} z}{d t^{q_{3}}}=x y-\frac{(8+\alpha) z}{3},
\end{aligned}
$$

where $d^{q_{i}} / d t^{q_{i}}=D_{*}^{q_{i}}(i=1,2,3)$. Its order is denoted by $q=$ $\left(q_{1}, q_{2}, q_{3}\right)$ subject to $0<q_{1}, q_{2}, q_{3} \leq 1$, and $\alpha \in[0,1]$. Some examples of the chaotic attractor of system (26) with $q_{1}=$ $q_{2}=q_{3}=0.95$ are shown in Figures 1 and 2 .

Two systems in synchronization are called the master system and the slave system, respectively. From (26), the master system and the slave system can be expressed, respectively, as

$$
\begin{aligned}
& \frac{d^{q_{1}} x_{1}}{d t^{q_{1}}}=(25 \alpha+10)\left(x_{2}-x_{1}\right) \\
& \frac{d^{q_{2}} x_{2}}{d t^{q_{2}}}=(28-35 \alpha) x_{1}-x_{1} x_{3}+(29 \alpha-1) x_{2} \longrightarrow
\end{aligned}
$$

master system

$$
\begin{aligned}
& \frac{d^{q_{3}} x_{3}}{d t^{q_{3}}}=x_{1} x_{2}-\frac{(8+\alpha) x_{3}}{3}, \\
& \frac{d^{q_{1}} y_{1}}{d t^{q_{1}}}=(25 \alpha+10)\left(y_{2}-y_{1}\right)+u_{1} \\
& \frac{d^{q_{2}} y_{2}}{d t^{q_{2}}}=(28-35 \alpha) y_{1}-y_{1} y_{3}+(29 \alpha-1) y_{2}+u_{2} \longrightarrow
\end{aligned}
$$

slave system

$$
\frac{d^{q_{3}} y_{3}}{d t^{q_{3}}}=y_{1} y_{2}-\frac{(8+\alpha) y_{3}}{3}
$$

where $u_{1}, u_{2}$ are the control signals used to drive the slave system to follow the master system.

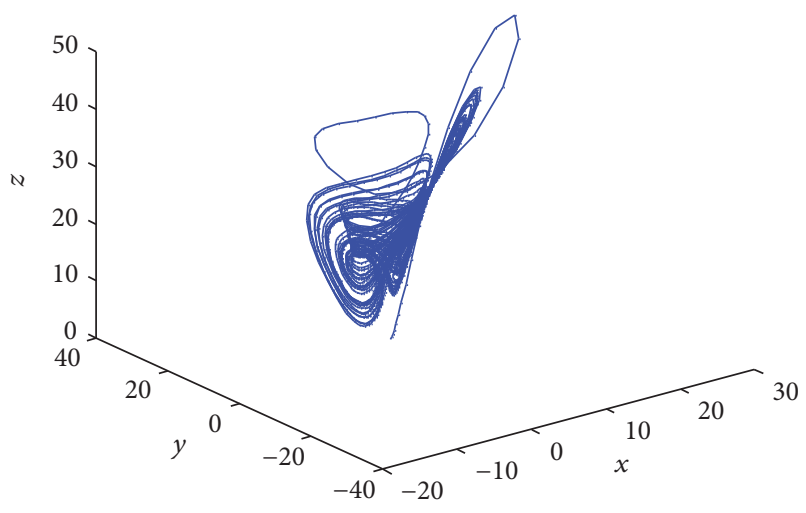

FIGURE 2: The fractional-order unified chaotic system with $q_{1}=q_{2}=$ $q_{3}=0.95$ and $\alpha=1$.

Denote the synchronization error as $e=y-x$. Our aim is to design a controller $u(t)=\left(u_{1}, u_{2}\right)^{T}$ such that controlled system (28) asymptotically synchronizes master system (27) in the sense that

$$
\lim _{t \rightarrow \infty}\|e\|=\lim _{t \rightarrow \infty}\left\|y\left(t, y_{0}\right)-x\left(t, x_{0}\right)\right\|=0
$$

Subtracting (27) from (28), then the synchronization error equation can be obtained as

$$
\begin{aligned}
\frac{d^{q_{1}} e_{1}}{d t^{q_{1}}}= & (25 \alpha+10)\left(e_{2}-e_{1}\right)+u_{1} \\
\frac{d^{q_{2}} e_{2}}{d t^{q_{2}}}= & (28-35 \alpha) e_{1}-e_{1} e_{3}-e_{1} x_{3}-e_{3} x_{1} \\
& \quad+(29 \alpha-1) e_{2}+u_{2} \\
\frac{d^{q_{3}} e_{3}}{d t^{q_{3}}}= & e_{1} e_{2}+e_{1} x_{2}+e_{2} x_{1}-\frac{(8+\alpha) e_{3}}{3} .
\end{aligned}
$$

Here, we take two steps to design a linear controller to globally asymptotically stabilize error system (30).

Step 1. From the proof process of Theorem 5 we know that if the matrix $A(\cdot)$ satisfies assumption (3) in Theorem 5 , system (15) is asymptotically stable. If we can design a controller $u_{1}$ such that it has a similar form to (15), $e_{1}$ will be stable. Let $u_{1}=-(25 \alpha+10) e_{2}$ and the first subsystem of (28) becomes

$$
\frac{d^{q_{1}} e_{1}}{d t^{q_{1}}}=-(25 \alpha+10) e_{1} .
$$

Obviously, for each $\alpha \in[0,1]$, it is globally asymptotically stable at $e_{1}=0$.

Step 2. A similar idea to $u_{1}$ is used in the design of the controller $u_{2}$. Let $u_{2}=-k e_{2}(k>29 \alpha-1)$. Consider the 
remaining subsystem of (30) and substitute $u_{2}=-k e_{2}(k>$ $29 \alpha-1)$ into the remaining subsystem. Then we can have

$$
\begin{aligned}
\frac{d^{q_{2}} e_{2}}{d t^{q_{2}}}= & (28-35 \alpha) e_{1}-e_{1} e_{3}-e_{1} x_{3}-e_{3} x_{1} \\
& +(29 \alpha-1) e_{2}+k e_{2} \\
\frac{d^{q_{3}} e_{3}}{d t^{q_{3}}}= & e_{1} e_{2}+e_{1} x_{2}+e_{2} x_{1}-\frac{(8+\alpha) e_{3}}{3} .
\end{aligned}
$$

Equation (32) can be rewritten by the matrix form as

$$
\left[\begin{array}{l}
d^{q_{2}} e_{2} \\
d^{q_{3}} e_{3}
\end{array}\right]=A\left[\begin{array}{l}
e_{2} \\
e_{3}
\end{array}\right]+B e_{1},
$$

where

$$
\begin{aligned}
& A=\left[\begin{array}{cc}
k+29 \alpha-1 & -e_{1}-x_{1} \\
e_{1}+x_{1} & -\frac{(8+\alpha)}{3}
\end{array}\right], \\
& B=\left[\begin{array}{c}
28-35 \alpha \\
0
\end{array}\right]+\left[\begin{array}{c}
-x_{3} \\
x_{2}
\end{array}\right] .
\end{aligned}
$$

Because master system (27) is a chaotic system, its states are bounded. This means that there exists a positive constant $\lambda>$ 0 such that

$$
\left|x_{i}\right| \leq \lambda \quad(i=1,2,3) .
$$

So the matrix $B$ is also a bounded matrix. Obviously, $A(x)$ has a special form that satisfies assumption (3) in Theorem 5. Form Theorem 5, error system (30) is globally asymptotically stable at $E(0,0,0)$, and slave system (28) will synchronize with master system (27).

3.2. Robustness Analysis. In order to verify the robustness of the linear chaos controller, the disturbance signal is added to the fractional-order unified chaotic system. Master system (27) and slave system (28) can be rewritten as the following forms:

$$
\begin{aligned}
\frac{d^{q_{1}} x_{1}}{d t^{q_{1}}}= & (25 \alpha+10) x_{2}-\left(25 \alpha+10+\Delta_{1}\right) x_{1} \\
\frac{d^{q_{2}} x_{2}}{d t^{q_{2}}}= & \left(28-35 \alpha+\Delta_{2}\right) x_{1}-\left(x_{1}+\Delta_{3}\right) x_{3} \\
& +\left(29 \alpha-1+\Delta_{4}\right) x_{2} \\
\frac{d^{q_{3}} x_{3}}{d t^{q_{3}}}= & \left(x_{1}+\Delta_{3}\right) x_{2}+\Delta_{5} x_{1}-\frac{\left(8+\alpha+\Delta_{6}\right) x_{3}}{3}, \\
\frac{d^{q_{1}} y_{1}}{d t^{q_{1}}=} & (25 \alpha+10) y_{2}-\left(25 \alpha+10+\Delta_{1}\right) y_{1}+u_{1} \\
\frac{d^{q_{2}} y_{2}}{d t^{q_{2}}}= & \left(28-35 \alpha+\Delta_{2}\right) y_{1}-\left(y_{1}+\Delta_{3}\right) y_{3} \\
& +\left(29 \alpha-1+\Delta_{4}\right) y_{2}+u_{2} \\
\frac{d^{q_{3}} y_{3}}{d t^{q_{3}}}= & \left(y_{1}+\Delta_{3}\right) y_{2}+\Delta_{5} y_{1}-\frac{\left(8+\alpha+\Delta_{6}\right) y_{3}}{3}
\end{aligned}
$$

where $\Delta_{i}(i=1,2, \ldots, 6)$ express the independent uncertainties which may be time varying or state dependent but are bounded by a constant $\gamma>0$ :

$$
\sup \left|\Delta_{i}\right| \leq \gamma \quad(i=1,2, \ldots, 6) .
$$

Form system (36), we can see that the system model can denote system uncertainties and parameter uncertainties.

Subtract (36) from (37) and we can get the following error system:

$$
\begin{aligned}
\frac{d^{q_{1}} e_{1}}{d t^{q_{1}}=} & (25 \alpha+10) e_{2}-\left(25 \alpha+10+\Delta_{1}\right) e_{1}+u_{1} \\
\frac{d^{q_{2}} e_{2}}{d t^{q_{2}}}= & \left(28-35 \alpha+\Delta_{2}\right) e_{1}-x_{3} e_{1} \\
& -\left(e_{1}+x_{1}+\Delta_{3}\right) e_{3}+\left(29 \alpha-1+\Delta_{4}\right) e_{2} \\
& +u_{2} \\
\frac{d^{q_{3}} e_{3}}{d t^{q_{3}}=} & \Delta_{5} e_{1}+x_{2} e_{1}+\left(e_{1}+x_{1}+\Delta_{3}\right) e_{2} \\
& -\frac{\left(8+\alpha+\Delta_{6}\right) e_{3}}{3} .
\end{aligned}
$$

Substitute $u_{1}=-(25 \alpha+10) e_{2} u_{2}=-k e_{2}(k>29 \alpha-1)$ into system (39) and we can get a matrix form as

$$
\begin{gathered}
\frac{d^{q_{1}} e_{1}}{d t}=-\left(25 \alpha+10+\Delta_{1}\right) e_{1} \\
{\left[\begin{array}{l}
\frac{d^{q_{2}} e_{2}}{d t^{q}} \\
\frac{d^{q_{3}} e_{3}}{d t^{q}}
\end{array}\right]=A^{\prime}\left[\begin{array}{l}
e_{2} \\
e_{3}
\end{array}\right]+B^{\prime} e_{1},}
\end{gathered}
$$

where $A^{\prime}=\left[\begin{array}{cc}k+29 \alpha-1+\Delta_{4} & -\left(e_{1}+x_{1}+\Delta_{3}\right) \\ e_{1}+x_{1}+\Delta_{3} & -\left(8+\alpha+\Delta_{6}\right) / 3\end{array}\right], B^{\prime}=\left[\begin{array}{c}28-35 \alpha+\Delta_{2} \\ \Delta_{5}\end{array}\right]+$ $\left[\begin{array}{c}-x_{3} \\ x_{2}\end{array}\right]$

When $k+29 \alpha-1+\Delta_{4}<0,8+\alpha+\Delta_{6}>0$, together with Theorem 5 , the error system (39) is globally asymptotically stable at $E(0,0,0)$. Hence the chaos controller is robust when $k+29 \alpha-1+\Delta_{4}<0,8+\alpha+\Delta_{6}>0$.

\section{Experimental Analyses}

In this section, the predictor-corrector method is used to obtain the solutions of fractional-order differential equations with step size 0.001 . If the step size is too large, the simulation results will not converge. Many authors in other references have adopted the step size as 0.001. And we have also adopted variable step size to do the simulation and we found the simulation results were better. In the simulation process for the fractional-order unified system, we let $q_{1}=$ $q_{2}=q_{3}=0.95$ and choose the initial states of the master and slave system as $\left(x_{1}(0), x_{2}(0), x(0)\right)^{T}=(1,2,3)^{T}$, $\left(y_{1}(0), y_{2}(0), y(0)\right)^{T}=(-1,-2,6)^{T}$.

When $\alpha=0$ system (26) is a fractional-order Lorenz chaotic system. When $\alpha=0.8$ system (26) is 


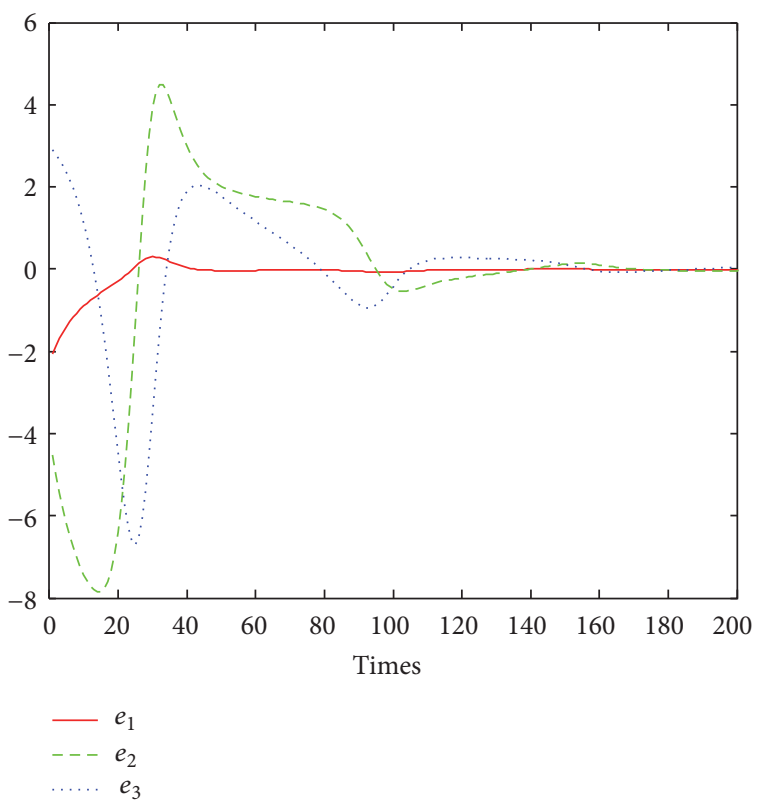

FIGURE 3: Synchronization errors of the fractional-order Lorenz system $(\alpha=0)$.

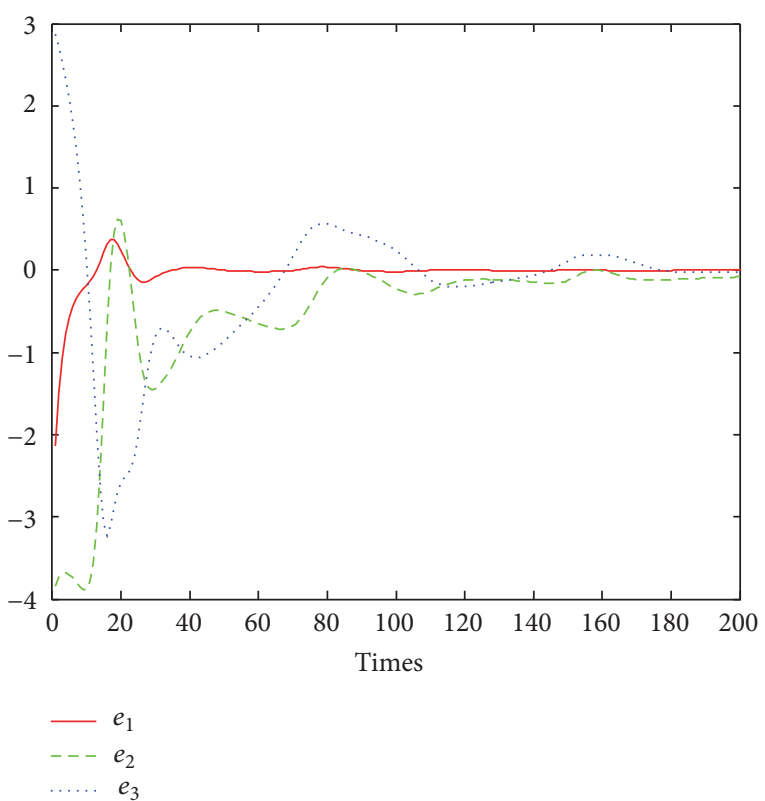

FIGURE 4: Synchronization errors of the fractional-order Lü system $(\alpha=0.8)$.

a fractional-order Lü chaotic system and when $\alpha=1$ system (26) is a fractional-order Chen chaotic system. The simulation results for synchronization of the fractionalorder Lorenz, Lü, and Chen chaotic systems with known parameters are shown in Figures 3-5, respectively. We chose the uncertain parameters $\left(\Delta_{1}, \Delta_{2}, \Delta_{3}, \Delta_{4}, \Delta_{5}, \Delta_{6}\right)^{T}=$ $\left(\sin x_{1}, 2 \cos x_{2}, \sin x_{3}, \cos y_{1}, \sin y_{2}, 1\right)^{T}$. Figures 6-8 show the synchronization of two identical Chen chaotic systems with uncertain parameters. As expected, one can see that

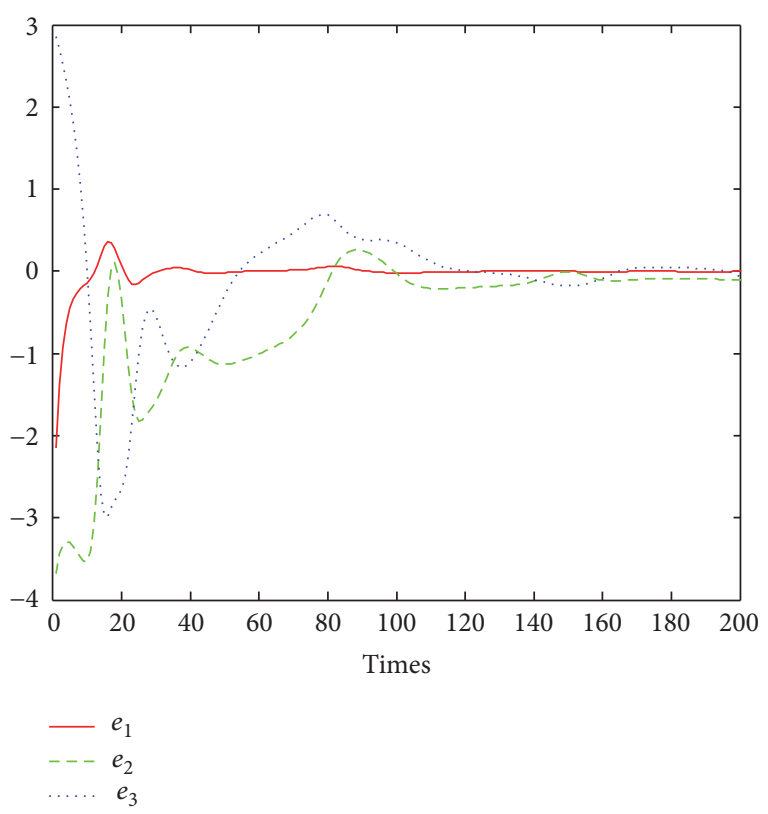

FIGURE 5: Synchronization errors of the fractional-order Chen system $(\alpha=1)$.

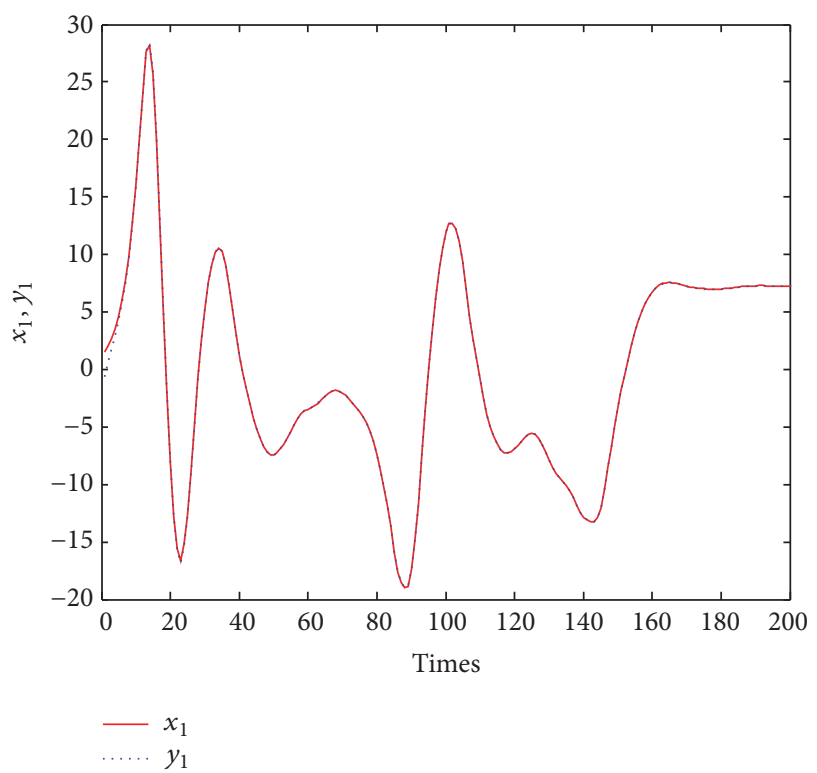

FIGURE 6: State trajectories of an uncertain fractional-order Chen system $x_{1}-y_{1}(\alpha=1)$.

the trajectories of the closed loop slave system can synchronize the trajectories of the master system. These results of the simulation verify the effectiveness of the proposed scheme.

\section{Conclusions}

This paper discusses a linear control scheme for synchronizing a class of cascade fractional-order chaotic systems. Based on continuous Lyapunov equation, the stability of the closed loop system is proved. This synchronization approach 


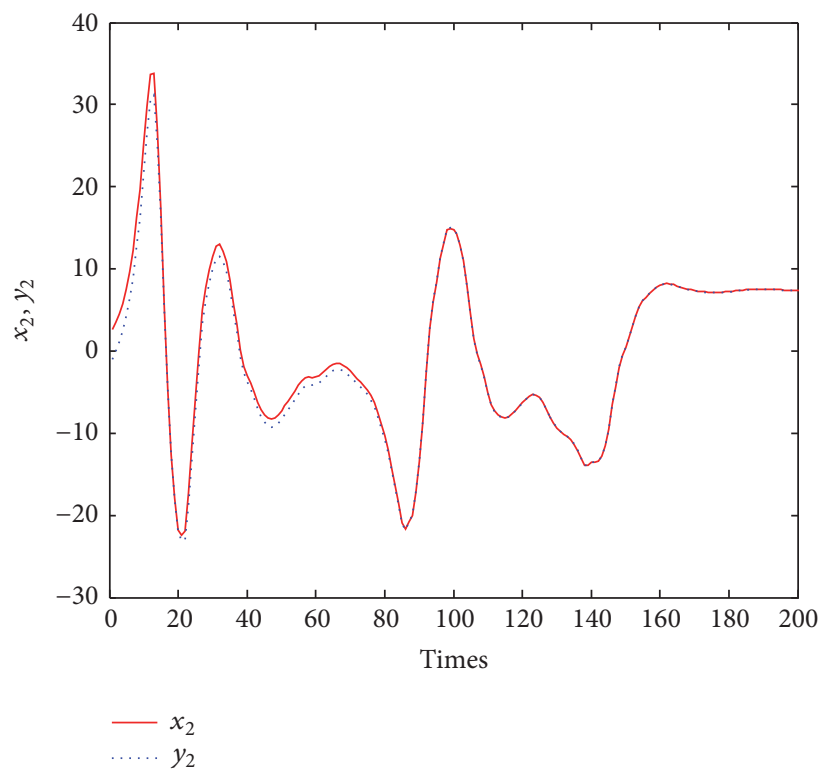

FIGURE 7: State trajectories of an uncertain fractional-order Chen system $x_{2}-y_{2}(\alpha=1)$.

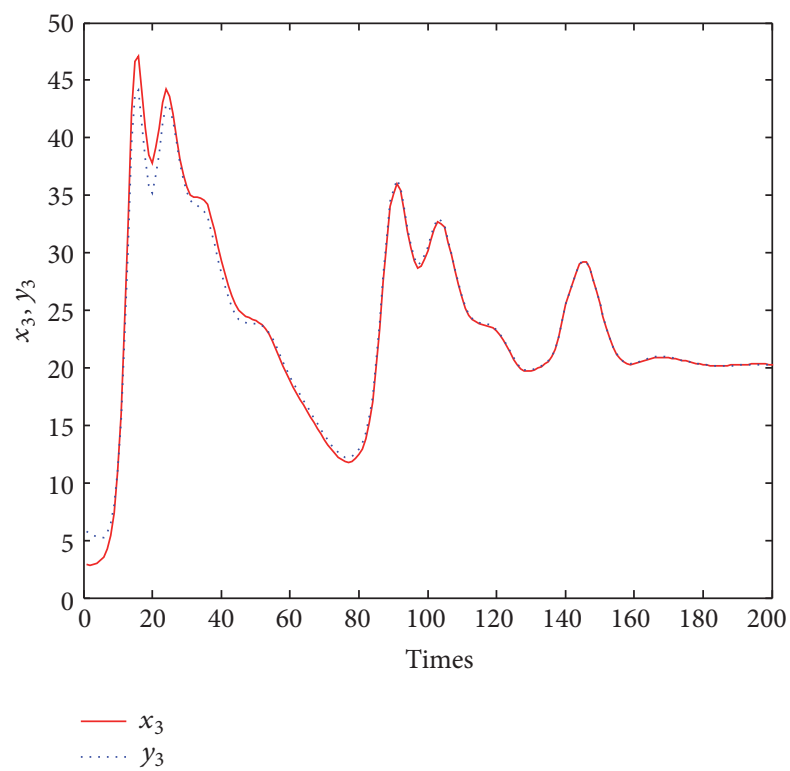

FIGURE 8: State trajectories of an uncertain fractional-order Chen system $x_{3}-y_{3}(\alpha=1)$.

is simple, global, and theoretically rigorous. Numerical simulations have been used to clarify the effectiveness of the proposed control laws. It should be noted that the introduced fractional linear controller is applicable for a large class of commensurate fractional-order chaotic systems in the presence of model uncertainties and eternal disturbances.

\section{Competing Interests}

The authors declare that they have no competing interests.

\section{Acknowledgments}

This work was supported by National Natural Science Foundation of China $(51375293,31570998)$ and by Science and Technology Commission of Shanghai Municipality Project (16511108600).

\section{References}

[1] I. Podlubny, Fractional Differential Equations, Academic Press, San Diego, Calif, USA, 1999.

[2] A. A. Kilbas, H. H. Srivastava, and J. J. Trujillo, Theory and Applications of Fractional Differential Equations, vol. 204 of North-Holland Mathematics Studies, 2006.

[3] K. Diethelm, The Analysis of Fractional Differential Equations, vol. 2004 of Lecture Notes in Mathematics, Springer, Berlin, Germany, 2010.

[4] I. Petras, Fractional-Order Nonlinear Systems: Modeling, Analysis and Simulation, Nonlinear Physical Science, Springer, New York, NY, USA, 2011.

[5] D. Baleanu, K. Diethelm, E. Scalas, and J. J. Trujillo, Fractional Calculus: Models and Numerical Methods, vol. 3 of Series on Complexity, Nonlinearity and Chaos, World Scientific, River Edge, NJ, USA, 2012.

[6] F. C. Meral, T. J. Royston, and R. Magin, "Fractional calculus in viscoelasticity: an experimental study," Communications in Nonlinear Science and Numerical Simulation, vol. 15, no. 4, pp. 939-945, 2010.

[7] Y. Luo, Y. Chen, and Y. Pi, "Experimental study of fractional order proportional derivative controller synthesis for fractional order systems," Mechatronics, vol. 21, no. 1, pp. 204-214, 2011.

[8] C. S. Drapaca and S. Sivaloganathan, "A fractional model of continuum mechanics," Journal of Elasticity, vol. 107, no. 2, pp. 105-123, 2012.

[9] W. H. Deng and C. P. Li, "Chaos synchronization of the fractional Lü system," Physica A: Statistical Mechanics and Its Applications, vol. 353, no. 1, pp. 61-72, 2005.

[10] J. G. Lu and G. Chen, "A note on the fractional-order Chen system," Chaos, Solitons \& Fractals, vol. 27, no. 3, pp. 685-688, 2006.

[11] X.-Y. Wang, Y.-J. He, and M.-J. Wang, "Chaos control of a fractional order modified coupled dynamos system," Nonlinear Analysis, Theory, Methods \& Applications, vol. 71, no. 12, pp. 6126-6134, 2009.

[12] V. Daftardar-Gejji and S. Bhalekar, "Chaos in fractional ordered Liu system," Computers \& Mathematics with Applications, vol. 59, no. 3, pp. 1117-1127, 2010.

[13] S. Kuntanapreeda, "Robust synchronization of fractional-order unified chaotic systems via linear control," Computers \& Mathematics with Applications, vol. 63, no. 1, pp. 183-190, 2012.

[14] E. N. Lorenz, "Deterministic nonperiodic flow," Journal of the Atmospheric Sciences, vol. 20, no. 2, pp. 130-141, 1963.

[15] E. Ott, C. Grebogi, and J. A. Yorke, "Controlling chaos," Physical Review Letters, vol. 64, no. 11, pp. 1196-1199, 1990.

[16] A. S. Hegazi, E. Ahmed, and A. E. Matouk, "On chaos control and synchronization of the commensurate fractional order Liu system," Communications in Nonlinear Science \& Numerical Simulation, vol. 18, no. 5, pp. 1193-1202, 2013.

[17] J. Hao, X. Xiong, H. Bin, and N. Sun, "Controlling chaos for fractional order loss type of coupled dynamos systems via 
feedback," International Journal of Bifurcation and Chaos, vol. 25, no. 9, Article ID 1550111, 2015.

[18] A. Razminia, V. J. Majd, and D. Baleanu, "Chaotic incommensurate fractional order Rössler system: active control and synchronization," Advances in Difference Equations, vol. 2011, no. 1, pp. 1-12, 2011.

[19] M.-F. Danca and R. Garrappa, "Suppressing chaos in discontinuous systems of fractional order by active control," Applied Mathematics and Computation, vol. 257, pp. 89-102, 2015.

[20] M. R. Faieghi, S. Kuntanapreeda, H. Delavari, and D. Baleanu, "LMI-based stabilization of a class of fractional-order chaotic systems," Nonlinear Dynamics, vol. 72, no. 1-2, pp. 301-309, 2013.

[21] D.-Y. Chen, Y.-X. Liu, X.-Y. Ma, and R.-F. Zhang, "Control of a class of fractional-order chaotic systems via sliding mode," Nonlinear Dynamics, vol. 67, no. 1, pp. 893-901, 2012.

[22] C. Yin, S.-M. Zhong, and W.-F. Chen, "Design of sliding mode controller for a class of fractional-order chaotic systems," Communications in Nonlinear Science and Numerical Simulation, vol. 17, no. 1, pp. 356-366, 2012.

[23] D. Matignon, "Stability results for fractional differential equations with applications to control processing," in Proceedings of the IMACS IEEE-SMC, pp. 963-968, Lille, France, 1996. 


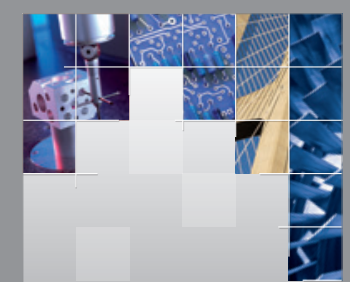

\section{Enfincering}
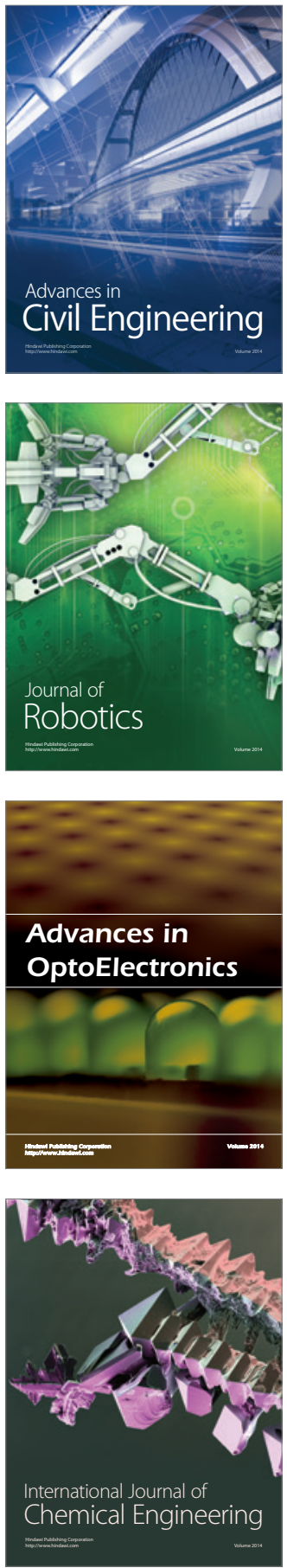

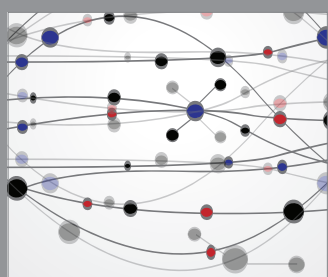

The Scientific World Journal

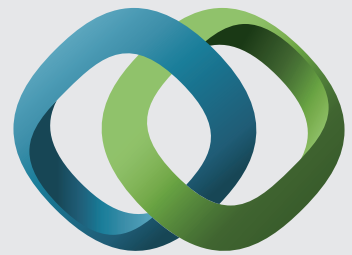

\section{Hindawi}

Submit your manuscripts at

http://www.hindawi.com
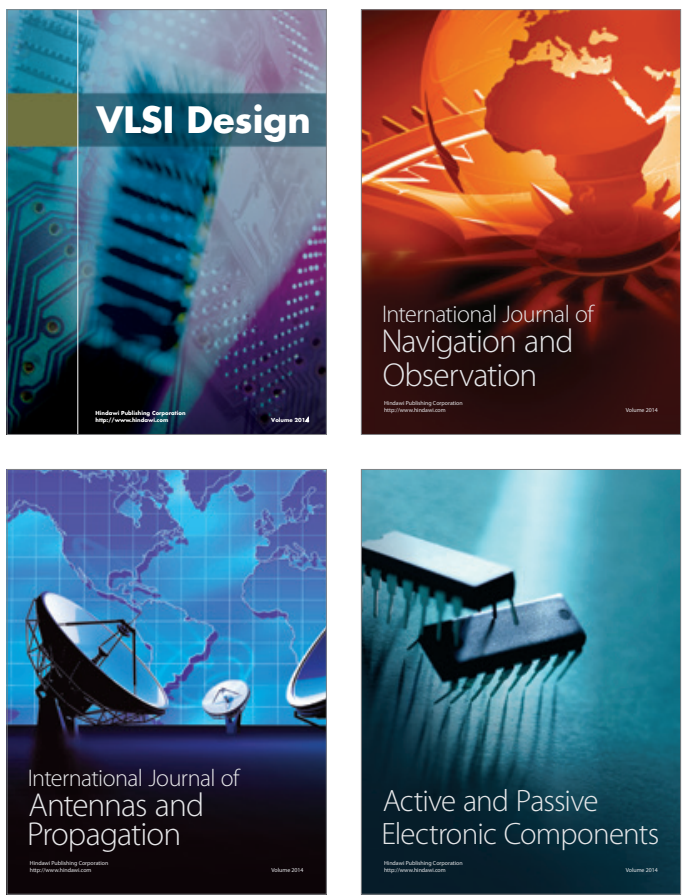
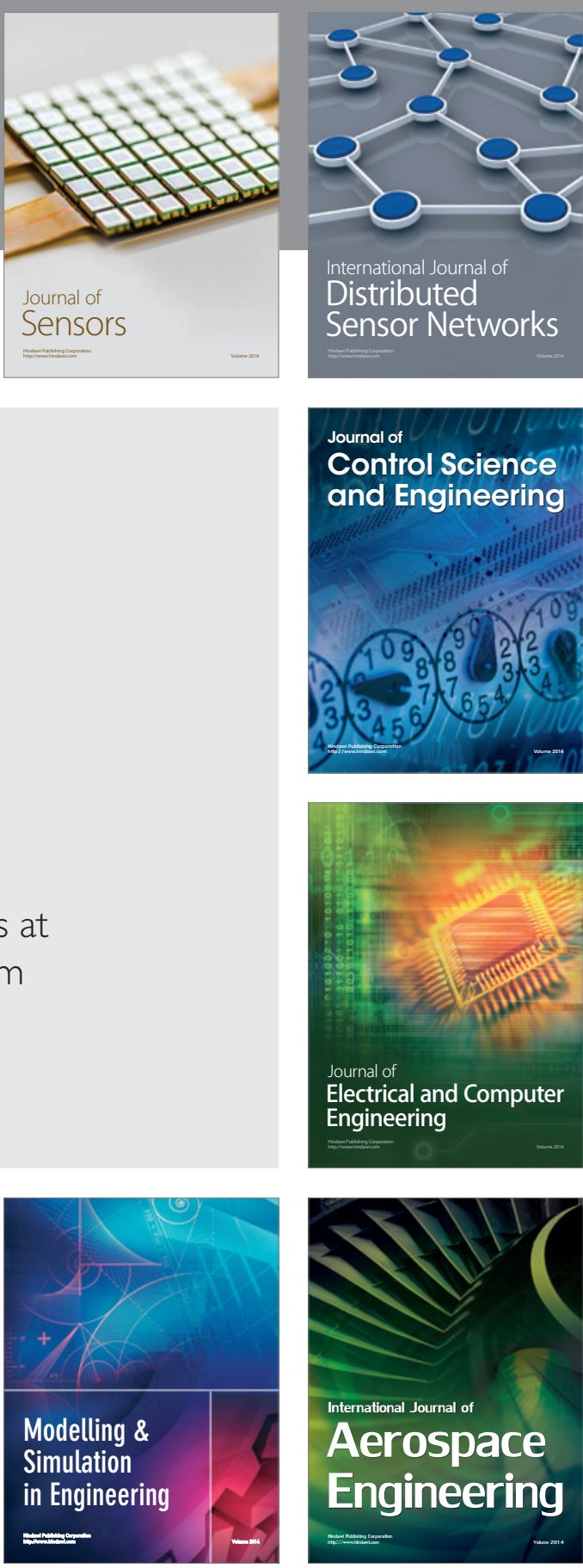

International Journal of

Distributed

Sensor Networks

Journal of

Control Science

and Engineering
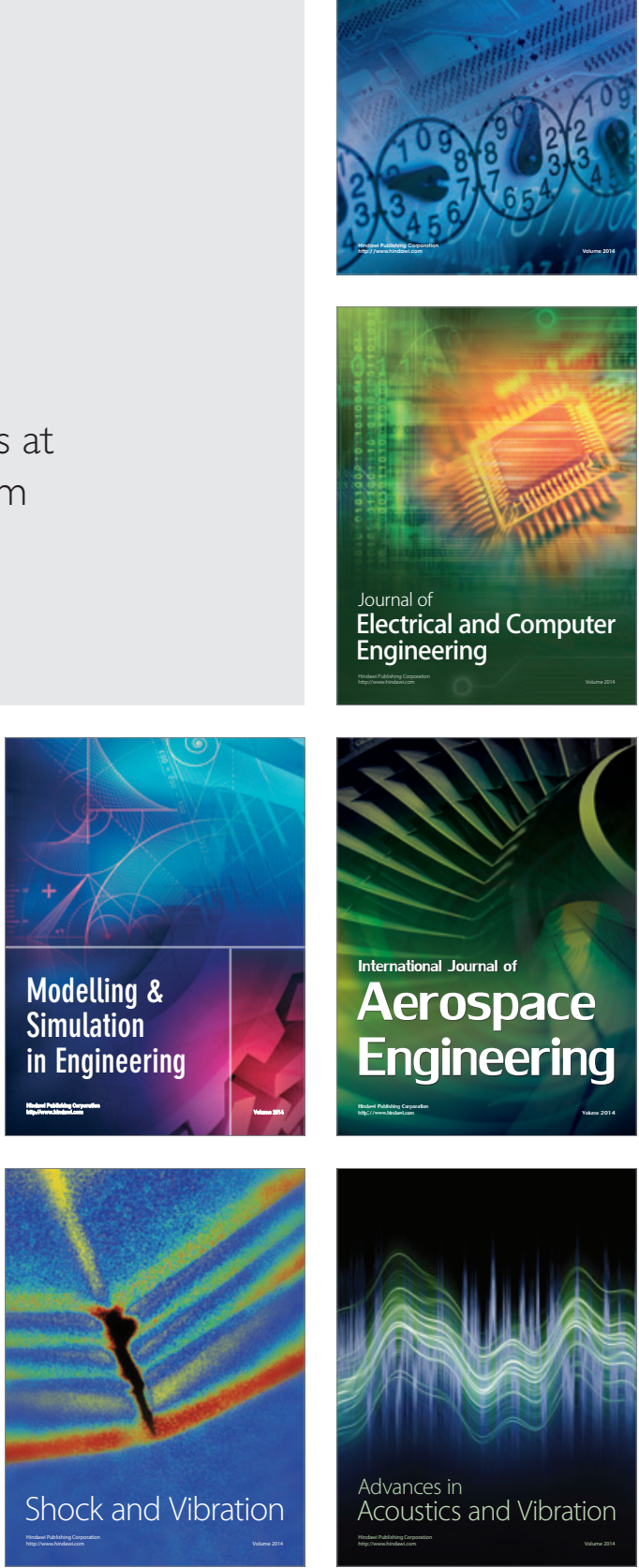